\title{
Fatty-acid amide hydrolase polymorphisms and post- traumatic stress disorder after penetrating brain injury
}

\author{
M Pardini ${ }^{1,2,3}$, F Krueger ${ }^{4,5}$, M Koenigs ${ }^{6}$, V Raymont ${ }^{7,8}$, C Hodgkinson $^{9}$, S Zoubak ${ }^{9}$, D Goldman ${ }^{9}$ and J Grafman ${ }^{1,10}$
}

The past few years have seen an increase in the clinical awareness of post-traumatic stress disorder (PTSD), one of the most disabling and least understood behavioral disorders. Although the biological bases of PTSD are poorly understood, fatty-acid amide hydrolase (FAAH) activity has been linked with arousability and aversive-memories extinction, that is, two key features of PTSD. In this study, we investigated the association between the FAAH genetic polymorphisms and PTSD development and maintenance. We assessed PTSD frequency in a group of male Vietnam war veterans who suffered combat-related penetrating traumatic brain injury, that is, a relatively homogeneous population regarding the nature of the events that led to PTSD. We showed that rs2295633, a single-nucleotide polymorphism of FAAH, was significantly associated with PTSD diagnosis in subjects without lesions in the ventromedial prefrontal cortex. Moreover, the presence of the $C$ allele was associated with more severe re-experiencing of trauma and more negative reported childhood experiences. In conclusion, our data suggest that FAAH has an important role in PTSD through modulation of aversive memories and point to both a novel therapeutic target and a possible risk marker for this condition.

Translational Psychiatry (2012) 2, e75; doi:10.1038/tp.2012.1; published online 31 January 2012

\section{Introduction}

Post-traumatic stress disorder (PTSD) is a behavioral disorder characterized by recurrent re-experiencing of the trauma, avoidance of trauma reminders, and elevated arousal that develop after exposure to a traumatic event. ${ }^{1}$ One unresolved issue in our understanding of PTSD is the relationship between the exposure to a traumatic event and the development of the disorder. Indeed, although the lifetime risk of developing PTSD is around $8 \%,{ }^{2}$ the lifetime risk to be exposed to psychological traumatic events is much higher, ranging in different studies between 60 and $90 \% .^{2,3}$ Cognitive theories of PTSD point to a heightened sensibility to trauma and to an impairment of aversive memories extinction as two key factors of PTSD development and maintenance. ${ }^{4}$ Although the biological factors underlying PTSD are still poorly understood, recent data suggest that the brain endocannabinoid system has a role in this condition., 6 Indeed, in animal studies an increase of endocannabinoid transmission has been shown to improve extinction of conditioned fear to aversive stimuli and to reduce anxiety-related behaviors. ${ }^{7,8}$ Moreover, pharmacological modulation of endocannabinoid tone has been proposed as a possible target for anxiety spectrum disorders, such as PTSD. ${ }^{5}$

On the basis of this evidence, we investigated the association between PTSD development and maintenance and the genetic polymorphisms of fatty-acid amide hydrolase (FAAH). FAAH is an integral membrane enzyme that is primarily responsible for the degradation of the endocannabinoids in the brain. Moreover, its activity has been linked with aversive memory processing ${ }^{7}$ and arousability, ${ }^{6}$ two of the cognitive processes altered in PTSD. To shed light on the role of FAAH in PTSD, we assessed PTSD frequency in a group of male Vietnam war veterans, who suffered combat-related penetrating traumatic brain injury (PTBI). ${ }^{9}$ This population presents with some unique advantages such as the long follow-up period, the extensive cognitive and behavioral preinjury and post-injury medical data and the relative homogeneity regarding the nature of the events that led to PTSD development, that is, suffering PTBI during their service in Vietnam. ${ }^{9,10}$

\footnotetext{
${ }^{1}$ Cognitive Neuroscience Section, National Institute of Neurological Disorders and Stroke-National Institutes of Health, Bethesda, MD, USA; ${ }^{2}$ Department of Neurosciences, Ophthalmology and Genetics, University of Genoa, Genoa, Italy; ${ }^{3}$ Magnetic Resonance Research Centre on Nervous System Diseases, University of Genoa, Genoa, Italy; ${ }^{4}$ Department of Molecular Neuroscience, George Mason University, Fairfax, VA, USA; ${ }^{5}$ Department of Psychology, George Mason University, Fairfax, VA, USA; ${ }^{6}$ Department of Psychiatry, University of Wisconsin-Madison, Madison, WI, USA; ${ }^{7}$ Department of Radiology, Johns Hopkins University, Baltimore, MD, USA; ${ }^{8}$ Department of Medicine, Imperial College London, London, UK; ${ }^{9}$ Laboratory of Neurogenetics, National Institute on Alcohol Abuse and Alcoholism, National Institutes of Health, Bethesda, MD, USA; ${ }^{10}$ Traumatic Brain Injury Research Laboratory, Kessler Foundation Research Center, West Orange, NJ, USA

Correspondence: Dr J Grafman, Traumatic Brain Injury Research Laboratory, Kessler Foundation Research Center, 1199 Pleasant Valley Way West Orange, NJ 07052, USA or Dr F Krueger, Department of Molecular Neuroscience, Krasnow Institute for Advanced Study, 4400 University Drive, Mail Stop 2A1 George Mason University Fairfax, Fairfax, VA 22030, USA.

E-mail: jgrafman@kesslerfoundation.org or FKruger@gmu.edu

This article is dedicated to the memory of AdB.

Keywords: endocannabinoids; post-traumatic stress disorder; single-nucleotide polymorphisms

Received 3 November 2011; revised 8 December 2011; accepted 11 December 2011
} 


\section{Methods}

Subject enrollment and PTSD evaluation. Enrolled subjects were drawn from the Vietnam Head Injury Study (VHIS) Phase III registry as described elsewhere. ${ }^{11} \mathrm{We}$ conducted Phase III between 2003 and 2006 at Bethesda National Naval Medical Center (36-39 years post-injury). PTBI subjects enrolled in the VHIS registry were included in the study if they agreed to undergo genetic analysis for FAAH polymorphisms and if their medical history was negative for alcohol or substance-abuse disorder, dementia, mood or psychotic disorder as assessed by clinical data. Our experimental sample thus consisted of 115 male Caucasian Vietnam war veterans recruited from the VHIS registry. Each subject underwent neurological and psychiatric examinations and a non-contrast brain CT scan. Psychiatric examination was aimed to verify the presence of other behavioral disorders and to enquiry on early psychological negative experiences. The presence and extent of early negative experiences was evaluated with the Early Trauma Inventory $(\mathrm{ETI})$, clinician version, ${ }^{12}$ a semi-structured interview widely used to quantify negative life-events in childhood and adolescence Pre-injury characteristics and clinical follow-up data of the participants were available from military and Veterans Administration records. PTSD was evaluated using the Structured Clinical Interview for DSM-IV-TR Axis I disorders $^{13}$ and the Clinician-Administered PTSD Scale. ${ }^{14}$ All subjects gave informed consent to the study. Our institution ethics committee approved all the study procedures.

CT acquisition and analysis. Axial non-contrast CT scans were acquired on a GE Medical Systems Light Speed Plus CT scanner in helical mode. Images were reconstructed with an in-plane voxel size of $0.4 \mathrm{~mm} \times 0.4 \mathrm{~mm}$, overlapping slice thickness of $2.5 \mathrm{~mm}$ and a $1 \mathrm{~mm}$ slice interval. Lesion location and volume were determined from CT images by manual tracing using the Analysis of Brain Lesion (ABLe) ${ }^{15}$ software implemented in MEDx v3.44 (Medical Numerics) with enhancements to support the Automated Anatomical Labeling atlas. A trained neuropsychiatrist (VR) performed the tracings, which were then reviewed by an experienced observer (JG), who was blind to the results of the clinical evaluations. The skull and scalp components of the CT volume were then removed; each volume was spatially normalized to a de-skulled CT scan, which was previously spatially normalized to match the shape of the $\mathrm{T} 1 \mathrm{MNI}$ brain (standard of the International Consortium for Brain Mapping). The ABLe program was used to exclude the manually delineated lesion from the spatial normalization process to improve registration accuracy. Spatial normalization was performed using an automated image registration algorithm using a 12-parameter affine model on de-skulled CT scans. Ventromedial prefrontal cortex (vmPFC) territories were then identified on CT images according to a previous publication. ${ }^{9}$ In particular, a subject was included in the VMPFC group if he showed a lesion encompassing at least $15 \%$ of the vmPFC territory, otherwise he was included in the vmPFC lesion-free group. ${ }^{9}$
Genotyping. Enrolled subjects were genotyped for seven tagging single-nucleotide polymorphisms (SNPs) at FAAH (rs913168, rs6703669, rs3766246, rs324420, rs324419, rs2295633, rs7524148) included in a published genetic array as previously described. ${ }^{16}$

Statistical analysis. On the basis of our previous finding ${ }^{9}$ showing that lesions to the vmPFC significantly reduced PTSD prevalence, we analyzed the effect of FAAH SNP on PTSD separately for subjects with and without vmPFC lesions. In these experimental groups, the association between FAAH SNPs and PTSD diagnosis was evaluated using chi-square statistics. Differences both in PTSD symptomatology according to DSM-IV-TR PTSD clusters and in early psychological negative experiences were assessed with non-parametric tests for between FAAH rs2295633 C/- and T/T groups using Mann-Whitney $U$ tests. Statistical threshold was set at 0.05 (two-tailed, Bonferroni-corrected for multiple comparisons).

\section{Results}

FAAH SNPs and PTSD prevalence. First, we investigated whether any of the FAAH SNPs were associated with an increase in PTSD diagnosis separately for those subjects with and without vmPFC lesions, given the known role of vmPFC areas in modulating PTSD risk. ${ }^{9}$ In TPBI subjects without vmPFC lesions, rs2295633 was the only FAAH SNP to reveal a significant difference in PTSD prevalence between the ancestral/- vs variant/variant subjects (Supplementary Table 1). C/- (Ancestral/- subjects, compared with $\mathrm{T} / \mathrm{T}$ (Variant/Variant) subjects, presented with a higher PTSD diagnosis prevalence (20 PTSD + out of 43 subjects vs 5 PTSD + out of 37 subjects; $46.5 \%$ vs $13.5 \%$; chi-square $=10.1 ; P=0.001$; Figure 1 ).

This observed higher prevalence of PTSD diagnosis for the $\mathrm{C} /$ - subjects was not due to differences in cognitive deficits in other domains or demographic differences. They were

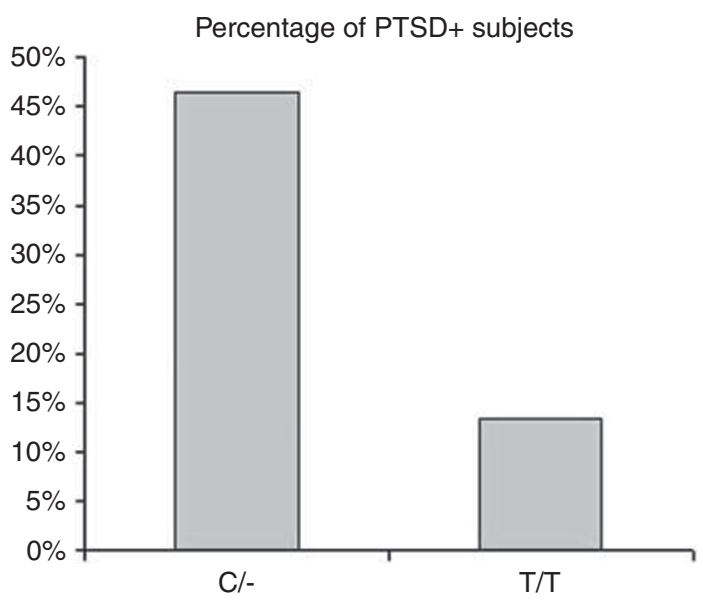

Figure 1 Prevalence of PTSD + subjects according to FAAH rs2295633 SNP genotype in those subjects with an intact vmPFC. 


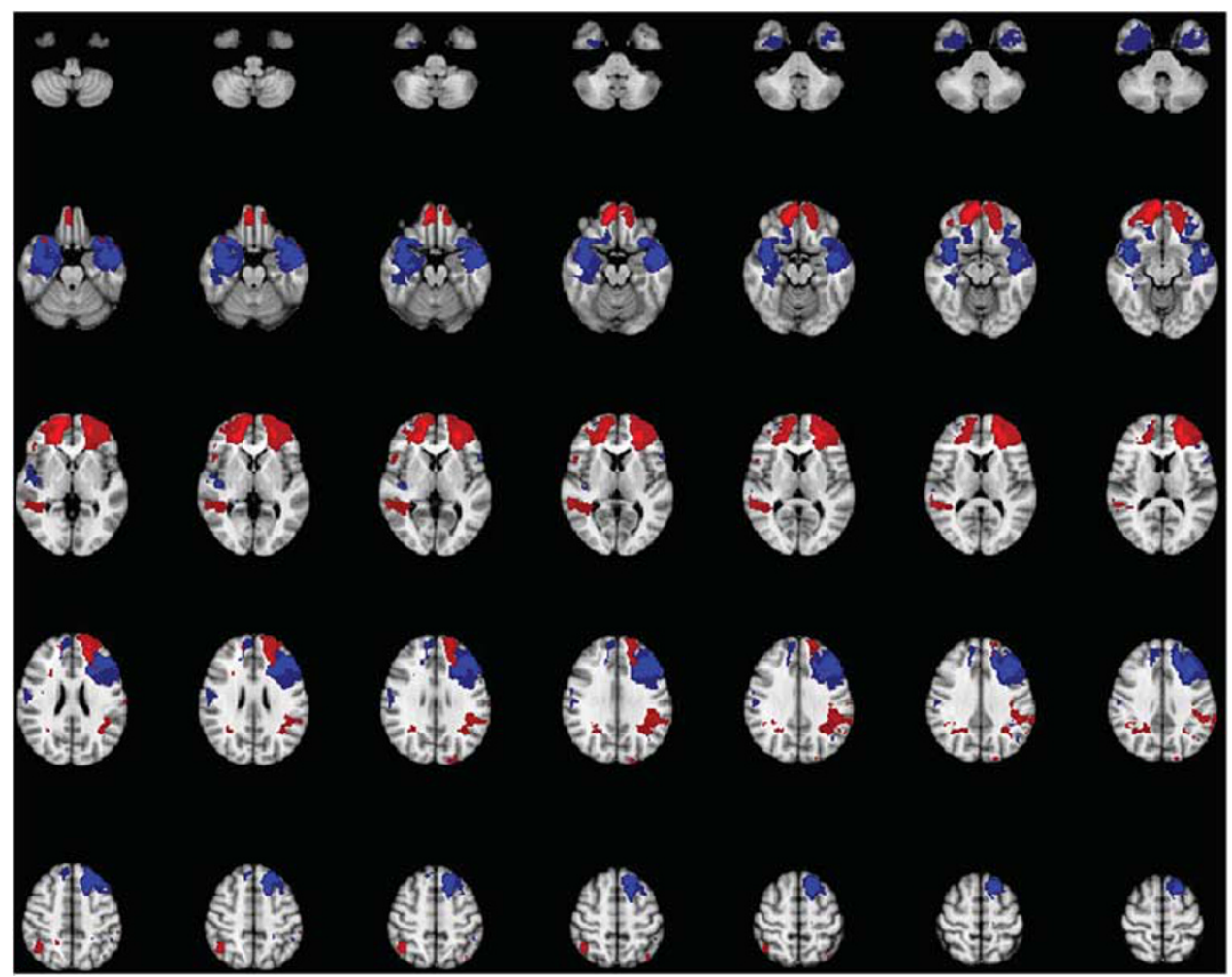

Figure 2 Lesions distribution map for the vmPFC lesion and for the vmPFC lesion-free groups. In red are shown those areas relatively more damaged in the vmPFC group compared with the vmPFC lesion-free group, whereas in blue are shown those areas relatively more damaged in the vmPFC lesion-free group compared with the vmPFC lesion group.

matched in age $(t=0.78 ; P=0.8)$ depressive levels (Beck Depression Inventory, 2nd edition) $(t=0.68 ; P=0.5),{ }^{17}$ preinjury intelligence (Armed Forces Qualification Test), $(t=0.72 ; P=0.6),{ }^{18}$ education $(t=0.82 ; P=0.6)$ emotional intelligence (Mayer-Salovey-Caruso Emotional Intelligence Test) $(t=0.42 ; P=0.8),{ }^{19}$ memory performance (Wechsler Memory Scale 3rd edition total score) $(t=0.65 ; P=0.8),{ }^{20}$ and percentage of total brain damage $(t=0.58 ; P=0.5)$ (Supplementary Table 2). Moreover, all the subjects included in the two aforementioned experimental groups were of Caucasian ethnicity.

No FAAH SNPs revealed any significant difference in PTSD prevalence between the ancestral/- vs variant/variant groups when analyzing only those subjects with vmPFC lesions (Supplementary Table 1). PTSD distribution in the vmPFC lesion group for the rs2295633 SNP was 3 subjects out of 20 for the C/- genotype and 3 subjects out of 13 for the T/T genotype (chi-square $=0.35 ; P=0.55$ ). Lesion distribution differences between the two experimental lesion groups are reported in Figure 2.

FAAH rs2295633 SNP and PTSD symptomatology. Given these results, the following analyses were limited to the rs2295633 SNP (C/- vs T/T). We thus examined the differences in PTSD clinical symptomatology between $\mathrm{C} /-$ and $\mathrm{T} / \mathrm{T}$ subjects starting with those without vmPFC damage. In this group, C/- subjects showed significantly more PTSD cluster 'B' symptoms according to the DSM-IV-
TR diagnostic criteria (persistent re-experiencing of the traumatic event) ${ }^{1}$ than $\mathrm{T} / \mathrm{T}$ subjects both taking into account only those patients with a PTSD clinical diagnosis ( $2.8 \pm 0.3$ vs $1.1 \pm 0.1 ; P=0.005 ; z=-2.73$; Figure 3 ) or the whole experimental group $(2.0 \pm 0.2$ vs $0.7 \pm 0.2 ; P=0.004$; $z=-2.86)$. There was no difference in the frequency of cluster 'C' (avoidance) (PTSD + subjects: $P=0.63$; $z=-0.4$. All subjects: $P=0.4 ; z=-0.8$ ) and cluster ' $D$ ' (arousal) (PTSD + subjects: $P=0.58 ; z=-0.6$. All subjects: $P=0.85 ; z=-0.78)$ symptoms between the two genotypes. Moreover, taking into account the whole sample of patients with vmPFC damage, there were no differences in frequency of cluster 'B' $(P=0.4 ; z=-0.9)$, 'C' $(P=0.6 ; z=-1.6)$, or 'D' $(P=0.5 ; z=-0.8)$ symptoms.

FAAH rs2295633 SNP and early experiences. Then, given the observed effect of FAAH SNP on re-experiencing of the traumatic event, we determined whether individual genetic differences in the FAAH gene were also linked with more intense recollections of negative childhood events. This analysis was run only in the group without vmPFC damage, given the observed low prevalence of PTSD in the vmPFC lesion group. Our data showed that in the C/- group PTSD + subjects presented with more severe recollections of early psychological trauma compared with PTSD - subjects (as assessed with the $\mathrm{ETI} ;{ }^{12} 6.35 \pm 0.8$ vs $2.0 \pm 0.4 ; z=-2.67$; $P=0.005$; Figure 3 ) in the absence of the differences in emotional aptitude (MSCEIT; $P=0.3$ ), total pre-injury 

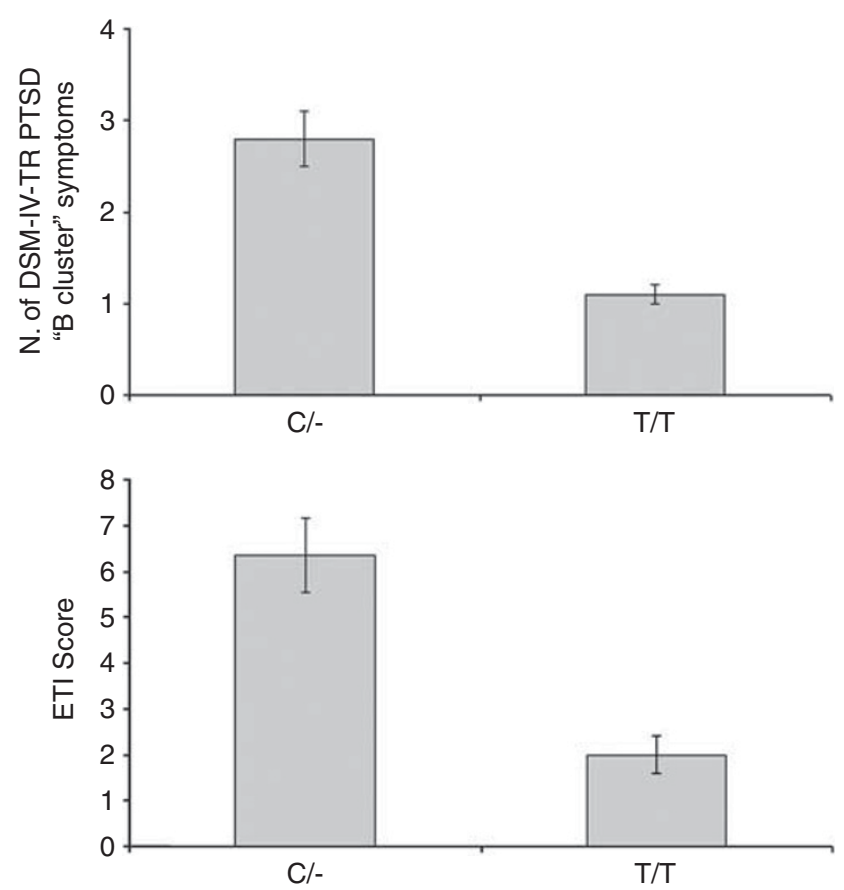

Figure 3 Upper panel: traumatic event re-experiencing symptomatology according to DSM-IV-TR (B 'Cluster' PTSD symptoms) distribution and according to FAAH rs2295633 SNP genotype in PTSD + subjects with intact vmPFC cortex. Lower panel: childhood negative events (ETI scale) distribution according to FAAH rs2295633 SNP genotype in PTSD + subjects with intact vmPFC cortex.

intelligence $(P=0.2)$ or memory scores $(P=0.5)$ between the two subgroups. In the $\mathrm{T} / \mathrm{T}$ group there was no difference in ETI scores between T/T PTSD + and T/T PTSD- subjects (5.6 \pm 1.2 vs $4.6 \pm 0.7 ; P=0.3$ ).

\section{Discussion}

Our findings suggest that a FAAH genetic polymorphism modulates PTSD risk in PTBI subjects with intact VmPFC but not in those subjects with vmPFC lesions. Presence of the wild-type allele at this SNP was linked with an increase of recollections of the traumatic event. Lastly, we showed that negative early experiences increased PTSD risk only in those subjects presenting at least one wild-type allele.

To date, the relationship between the presence of the $\mathrm{C}$ (that is wild-type allele) and FAAH function is not completely understood. Recent data from an amphetamine challenge study linked a heightened arousability to the $\mathrm{C}$ allele; increased arousal has been linked with reduced FAAH activity in animal models thus suggesting possible higher FAAH activity in $\mathrm{C}$ allele carriers compared with $\mathrm{T}$ allele carriers. ${ }^{6}$

Published data on FAAH activity showed that FAAH inhibition and thus increase of endocannabinoid levels has been linked with heightened aversive memory extinction and reduced anxiety levels in animal studies. ${ }^{7,8}$ Moreover reduction of endocannabinoid activity has been linked with impairments of extinction of aversive memories and increased anxiety levels. ${ }^{8}$
Our observation of an effect of FAAH only in those subjects with an intact vmPFC seems to be consistent with a role of vmPFC in traumatic events recollection and with our previous observation of a protective effect of vmPFC lesions on PTSD. ${ }^{9}$ Indeed in neuroimaging studies, recollection of traumatic events in PTSD subjects has been linked with vmPFC functions. ${ }^{19,20}$ Moreover aversive memory consolidation and extinction has been linked with prefrontal functions both in animal and human studies, ${ }^{21,22}$ thus damage to the neural structures linked with aversive memories extinction would likely overshadow the relatively more modest effects of a single SNP on consolidation and extinction processes. This observation is compatible with our previous report of the need of intact prefrontal structures to allow for genetic influences to modulate aggressive behaviors in PTBI. ${ }^{10}$

One of the major challenges in the current approach to PTSD is the identification of reliable markers for the factors that lead to an increased risk for developing the disorder (that is vulnerability) and of those that lead to a persistence of the disorder (that is maintenance). Identification of vulnerability markers could be a key factor in the development of primary prevention of PTSD in selected populations. Our data suggest that the FAAH rs2295633 SNP could serve as a possible marker for increased PTSD risk in subjects exposed to brain injury and combat experiences; future studies are warranted to explore the clinical utility of FAAH genotyping to guide primary prevention of PTSD in other at-risk populations. In addition, it would be helpful to understand the epigenetic/ environmental factors that might have led those patients with an increased risk not to develop PTSD.

Moreover, our data suggest that PTSD subjects presents different clinical and psychopathological characteristics according to FAAH genotype, with $\mathrm{C} /$ - subjects showing more intense recollections of traumatic events and of negative childhood experiences. Given the key role of extinction learning and of early experiences in different psychotherapeutic approaches to PTSD, FAAH genotyping could help to develop individualized therapies based on different genetic characteristics for the more severe and challenging cases of PTSD.

In conclusion, our findings point to $\mathrm{FAAH}$ as a contributor to PTSD after PTBI, possibly through the modulation of aversive memories by extinction processes. These data suggest a role for endocannabinoid signaling in the development and maintenance of PTSD and hint at the therapeutic potential of endocannabinoid systems-modulating drugs for PTSD patients. $^{5}$

\section{Conflict of interest}

Dr Goldman serves on the editorial boards of Biological Psychiatry and Addictions Biology. Dr Grafman serves as Co-editor of Cortex. All other authors declare no conflict of interest.

Acknowledgements. The work was supported by the US National Institute of Neurological Disorders and Stroke intramural research program and a project grant from the US Army Medical Research and Material Command administrated by the Henry M Jackson Foundation (Vietnam Head Injury Study Phase III: a 30-year post-injury follow-up study). 
Disclaimer. The views expressed in this article are those of the authors and do not necessarily reflect the official policy or position of the Department of the Navy, the Department of Defense, or the US Government.

1. American Psychiatric Association. Diagnostic and Statistical Manual of Mental Disorders, 4th edn, text revision. American Psychiatric Association: Washington, DC, 2000.

2. Kessler RC, Sonnega A, Bromet E, Hughes M, Nelson CB. Posttraumatic stress disorder in the National Comorbidity Survey. Arch Gen Psychiatry 1995; 52: 1048-1060.

3. Breslau N, Kessler RC, Chilcoat HD, Schultz LR, Davis GC, Andreski P. Trauma and posttraumatic stress disorder in the community: the 1996 Detroit Area Survey of Trauma. Arch Gen Psychiatry 1998; 55: 626-632.

4. Taylor S. Clinician's Guide to PTSD: A Cognitive-Behavioral Approach. The Guilford Press: New York, NY, 2006

5. Hill MN, Gorzalka BB. The endocannabinoid system and the treatment of mood and anxiety disorders. CNS Neurol Disord Drug Targets 2009; 8: 451-458.

6. Dlugos AM, Hamidovic A, Hodgkinson CA, Goldman D, Palmer AA, de Wit H. More aroused, less fatigued: fatty acid amide hydrolase gene polymorphisms influence acute response to amphetamine. Neuropsychopharmacology 2010; 35: 613-622.

7. Lutz B. The endocannabinoid system and extinction learning. Mol Neurobiol 2007; 36: 92-101.

8. Varvel SA, Wise LE, Niyuhire F, Cravatt BF, Lichtman AH. Inhibition of fatty-acid amide hydrolase accelerates acquisition and extinction rates in a spatial memory task. Neuropsychopharmacology 2007; 32: 1032-1041.

9. Koenigs M, Huey ED, Raymont V, Cheon B, Solomon J, Wassermann EM et al. Focal brain damage protects against post-traumatic stress disorder in combat veterans. Nat Neurosci 2008; 11: 232-237.

10. Pardini M, Krueger F, Hodgkinson C, Raymont V, Ferrier C, Goldman D et al. Prefrontal cortex lesions and MAO-A modulate aggression in penetrating traumatic brain injury Neurology 2011; 76: 1038-1045.

11. Raymont V, Greathouse A, Reding K, Lipsky R, Salazar A, Grafman J. Demographic, structural and genetic predictors of late cognitive decline after penetrating head injury. Brain 2008; 131(Part 2): 543-558.
12. Bremner JD, Vermetten $\mathrm{E}$, Mazure $\mathrm{CM}$. Development and preliminary psychometric properties of an instrument for the measurement of childhood trauma: the Early Trauma Inventory. Depress Anxiety 2000; 12: 1-12.

13. First MB, Spitzer RL, Gibbon M, Williams JB. Structured Clinical Interview for DSM-IV-TR Axis I Disorders, Research Version, Patient Edition (SCID-I/P). Biometrics Research: New York, NY, 2002.

14. Blake DD, Weathers FW, Nagy LM, Kaloupek DG, Gusman FD, Charney DS et al. The development of a clinician-administered PTSD scale. J Trauma Stress 1995; 8: 75-90.

15. Solomon J, Raymont V, Braun A, Butman JA, Grafman J. User-friendly software for the analysis of brain lesions (ABLe). Comput Methods Programs Biomed 2007; 86: 245-254.

16. Hodgkinson CA, Yuan Q, Xu K, Shen PH, Heinz E, Lobos EA et al. Addictions biology: haplotype-based analysis for 130 candidate genes on a single array. Alcohol Alcohol 2008; 43: 505-515.

17. Beck AT, Steer RA, Ball R, Ranieri W. Comparison of beck depression inventories-IA and II in psychiatric outpatients. J Pers Assess 1996; 67: 588-597.

18. Mayer JD, Salovey P, Caruso DR, Sitarenios G. Mayer-Salovey-Caruso Emotional Intelligence Test (Version 2.0) User's Manual. Multi-Health System: Toronto, Canada, 2002.

19. Wechsler D. WMS-III Administration and Scoring Manual. The Psychological Corporation: San Antonio, TX, 1997.

20. Bremner JD, Staib LH, Kaloupek D, Southwick SM, Soufer R, Charney DS. Neural correlates of exposure to traumatic pictures and sound in Vietnam combat veterans with and without posttraumatic stress disorder: a positron emission tomography study. Biol Psychiatry 1999; 45: 806-816.

21. Shin LM, Rauch SL, Pitman RK. Amygdala, medial prefrontal cortex, and hippocampal function in PTSD. Ann NY Acad Sci 2006; 1071: 67-79.

22. Quirk GJ, Beer JS. Prefrontal involvement in the regulation of emotion: convergence of rat and human studies. Curr Opin Neurobiol 2006; 16: 723-727.

Translational Psychiatry is an open-access journal published by Nature Publishing Group. This work is licensed under the Creative Commons Attribution-Noncommercial-No Derivative Works 3.0 Unported License. To view a copy of this license, visit http://creativecommons.org/licenses/by-nc-nd/3.0/

Supplementary Information accompanies the paper on the Translational Psychiatry website (http://www.nature.com/tp) 